Ophthalmologe 2014 · 111:1008-1009

DOI 10.1007/s00347-013-3008-6

Online publiziert: 10. Oktober 2014

(C) Springer-Verlag Berlin Heidelberg 2014

\section{Cursiefen}

Zentrum für Augenheilkunde, Universität zu Köln

\title{
Künstliche Hornhaut
}

\section{Aktuelle Versorgungsmöglichkeiten und zukünftige Perspektiven}

Die Transplantationschirurgie der Hornhaut macht im Moment einen rasanten Wandel durch. In den letzten Jahren fand eine „Revolution“ hin zu lamellären Operationsverfahren statt $[1,2]$. Inzwischen ist bei endothelialen Erkrankungen der Hornhaut wie der Fuchs-Endotheldystrophie und der pseudophaken bullösen Keratopathie ein lamelläres Operationsverfahren wie DMEK („Descemet membrane endothelial keratoplasty") oder DSAEK („Descemet stripping automated endothelial keratoplasty") der Goldstandard [1]. Auch bei Erkrankungen des vorderen Stromas wie dem Keratokonus werden immer häufiger lamelläre Verfahren mit geringerem Risikoprofil eingesetzt [2].

Im Gegensatz zu diesen erfreulichen Entwicklungen in avaskulären Normalrisikoaugen sind die Entwicklungen im Bereich der „vaskularisierten“ Hochrisikoaugen wesentlich geringer. Fortschritte wurde hier vor allem im Bereich der angioregressiven Therapie gegen vorbestehende Blutgefäße erzielt [3], des Weiteren im Bereich der HLA-Typisierung und der lokalen und systemischen Immunsupression $[4,5]$. Trotz all dieser Bemühungen verbleibt eine kleine, aber sehr schwer beeinträchtigte Gruppe von $\mathrm{Pa}$ tienten mit schwersten Erkrankungen der Augenoberfläche, denen auch mit einer allogenen HLA-typisierten Keratoplastik und einer systemischen Immunsupression nicht geholfen werden kann. Dies ist die „Nische“ der künstlichen Hornhaut (Keratoprothese).

Die Historie der Entwicklung von künstlichen Hornhäuten geht in das 19. Jahrhundert zurück. Auf diesem lan- gen Weg hin zu einem optimalen künstlichen Hornhautimplantat wurden verschiedenste Modelle ausgetestet und zum großen Teil auch wieder verworfen. Es gibt derzeit einige relativ häufig angewendete künstliche Hornhautvarianten, die sich weltweit im Einsatz bei schwersten Erkrankungen der Augenoberfläche bewährt haben. Dazu zählen die BostonKeratoprothese (Boston Kpro) und auch die OsteoOdontoKeratoProthese (OOKP; [6]). Für beide Verfahren gibt es zahlreiche publizierte Erfahrungen und mehrere Tausend weltweit durchgeführt Operationen z. B. bei der Boston Kpro. Gerade bei Patienten mit immunologisch vermittelten schwersten Erkrankungen der Augenoberfläche wie dem okulären Pemphigoid und dem Fuchs-Steven-Johnson-Syndrom, bei Patienten mit vaskularisierten Hochrisikoaugen nach mehrfachem Transplantatversagen und bei Patienten mit schwersten Beeinträchtigungen der Augenoberfläche nach Verätzung bieten diese Verfahren relativ zuverlässige Möglichkeiten einer zumindest zeitweiligen deutlichen Sehverbesserung [7]. Dennoch sind all diese Verfahren mit zahlreichen, zum Teil schwerwiegenden Komplikationen behaftet und erfordern eine intensive lebenslange Nachsorge sowie nicht selten Nachoperationen.

Ziel dieses Heftes ist es daher, zum einen den aktuellen Versorgungsstand mit den gängigen Keratoprothesen darzustellen. In dem Beitrag von Schrage et al. ["Aktuelle Versorgungsmöglichkeiten mit Keratoprothesen: Boston Kpro, Osteoodontokeratoprothese (OOKP) und KeraClear", in diesem Heft] werden die gängigen verfügbaren künstlichen Horn- hauttypen, die sich im klinischen Routineeingriff befinden, mit ihren Indikation, ihren Operationstechniken, den Komplikationsmöglichkeiten und den Nachsorgeprinzipien dargestellt.

\section{\) Große Hoffnungen \\ werden in die Entwicklung von Biocorneae gesetzt}

In Anbetracht der eben erwähnten reduzierten Langzeitergebnisse und möglichen Komplikationen bestehen Hoffnungen, mit der Entwicklung von besser bioverträglichen und zum Teil bioresorbierbaren Prothesen (Biocornea) die Prognose für die betroffenen Patienten zu verbessern [6]. Deshalb widmen sich 2 Beiträge dieses Themenheftes aktuellen Entwicklungen in diesem Bereich. Der Beitrag von Fuchsluger et al. („Neue Möglichkeiten der Augenoberflächenrekonstruktion mit Membranen aus Kollagen und biokompatiblen Elastomernanofasern“, in diesem Heft) stellt die Möglichkeiten der Entwicklung von künstlichen Hornhäuten basierend auf Kollagenlamellen dar. Der Beitrag von Hos et al. ["Dezellularisierte Kollagenmatrix aus der Schuppe des Tilapia-Fisches als Hornhautersatz (,BioCornea')“, in diesem Heft] zeigt die Möglichkeiten zur Entwicklung einer künstlichen Hornhaut mittels degradierbarem Fischkollagen als Ergebnis eines europäischen Forschungskonsortiums auf. Hier fördert die EU für 4 Jahre im Rahmen des COSTProgramms die europäische Zusammenarbeit zur Herstellung einer Biocornea (www.cost.eu/domains_actions/bmbs/ Actions/BM1302, www.biocornea.eu). 
Zusammenfassend gibt es derzeit zwar zuverlässige und anwendbare Therapiemöglichkeiten mit künstlichen Hornhäuten bei Patienten mit schwersten Erkrankungen der Augenoberfläche. In Anbetracht der Komplikationen und reduzierten Langzeitprognosen der Verfahren besteht jedoch große Hoffnung, mittels neuer Forschungsansätze hin zu „biologischeren“ künstlichen Hornhäuten die Langzeitprognose für diese schwer erkrankten Patienten längerfristig zu verbessern. Diese neuen Ansätze werden sich hoffentlich schon mittelfristig in der Klinik etablieren.

Ihnen eine anregende Lektüre!

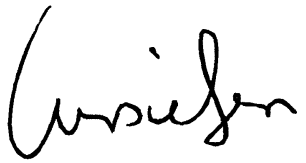

Claus Cursiefen

\section{Korrespondenzadresse}

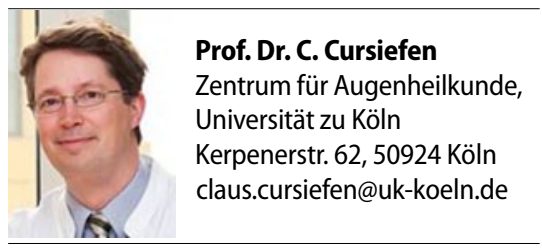

Interessenkonflikt. C. Cursiefen gibt an, dass kein Interessenkonflikt besteht.

\section{Literatur}

1. Maier P, Reinhard T, Cursiefen C (2013) Descemet stripping endothelial keratoplasty - rapid recovery of visual acuity. Dtsch Arztebl Int 110:365-371

2. Riss S, Heindl LM, Bachmann BO et al (2012) Pentacam-based big bubble deep anterior lamellar keratoplasty in patients with keratoconus. Cornea 31:627-632

3. Koenig Y, Bock F, Kruse FE et al (2012) Angioregressive pretreatment of mature corneal blood vessels before keratoplasty: fine-needle vessel coagulation combined with anti-VEGFs. Cornea 31:887-892

4. Lapp T, Reinhold D, Böhringer D, Reinhard T (2013) Human leukocyte antigen (HLA) system in ophthalmology. Ophthalmologe 110:849-861

5. Bock F, Maruyama K, Regenfuss B et al (2013) Novel anti(lymph)angiogenic treatment strategies for corneal and ocular surface diseases. Prog Retin Eye Res 34:89-124

6. Griffith M, Harkin DG (2014) Recent advances in the design of artificial corneas. Curr Opin Ophthalmol 25:240-247

7. Colby KA, Koo EB (2011) Expanding indications for the Boston keratoprosthesis. Curr Opin Ophthalmol 22:267-273

\section{Zelltransplantation lässt Mäuse bei Tageslicht wieder sehen}

Für das menschliche Sehen bei Tageslicht sind bestimmte Fotorezeptoren, die sogenannten Zapfen, verantwortlich. Die Degeneration dieser Zellen führt zu schweren Sehbehinderungen bei Krankheiten, wie der altersbedingten Makuladegeneration, der Zapfen-StäbchenDystrophie und Spätstadien der Retinitis Pigmentosa. Da die Netzhaut von Säugetieren, einschließlich der des Menschen, Fotorezeptoren selbst nicht ersetzen kann, führt das zum dauerhaften Verlust der Sehkraft. Bisher gibt es hier keine Heilungsmöglichkeiten.

Am DFG-Forschungszentrum für Regenerative Therapien Dresden - Exzellenzcluster an der TU Dresden (CRTD) ist es nun in Kooperation mit Forschern des Naturwissenschaftlichen und Medizinischen Institut (NMI), Universität Tübingen gelungen, zapfen-ähnliche Fotorezeptoren von Spendermäusen in die Netzhaut von Mäusen mit defektem Tageslichtsehen zu transplantieren.

Bei der Mehrzahl der Studien über Transplantationen von Fotorezeptoren bei Mäusen lag der Fokus bisher vor allem auf den Stäbchen-Fotorezeptoren. Stäbchen sind jedoch an schwache Lichtverhältnisse in der Dämmerung und der Nacht angepasst, sie dominieren daher die Netzhaut der nacht-aktiven Maus. Da im Gegensatz dazu das menschliche Sehen bei Tageslicht vor allem von ZapfenFotorezeptoren abhängt, nutzten die Wissenschaftler genetisch veränderte Mäuse, die ausschließlich Zapfen besitzen. Sie transplantierten aus diesen Mäusen isolierte zapfenähnliche Fotorezeptoren in Empfänger-Mäuse, deren Zapfen degeneriert, beziehungsweise nicht funktionsfähig, waren. Mit Hilfe elektrophysiologischer Messungen konnte erstmalig nachgewiesen werden, dass die transplantierten Zapfen funktional in die Netzhaut integrierten und auf hohe Lichtreize reagierten, die korrekt in der Empfängernetzhaut verarbeitet wurden.

Die Ergebnisse dieser Studie sind für die Entwicklung von Zellersatztherapien bei Erblindung von Bedeutung, da sie den ersten Nachweis liefern, dass sich die Sehkraft bei Tageslicht durch Zelltransplantation in die Säugetiernetzhaut prinzipiell wiederherstellen lässt.

Als nächstes gilt es, die Anzahl der transplantierten Zellen sowie deren Lebensdauer zu erhöhen und eine zuverlässige Quelle zur Gewinnung einer ausreichenden Anzahl von Spender-Fotorezeptoren für klinische Anwendungen zu etablieren. Dabei wird voraussichtlich die Entwicklung von Fotorezeptoren aus induziert pluripotenten und embryonalen Stammzellen für zukünftige Transplantationsstudien eine entscheidende Rolle spielen.

\section{Originalpublikation}

Tiago Santos-Ferreira, Kai Postel, Henrike Stutzki, Thomas Kurth, Günther Zeck, Marius Ader: Daylight vision repair by cell transplantation. Stem Cells, DOI: 10.1002/stem.1824.

Quelle: CRTD/DFG-Forschungszentrum für Regenerative Therapien Dresden - Exzellenzcluster an der TU Dresden (http://www.crt-dresden.de) 